\title{
Altered exosomal microRNA profiles in bronchoalveolar lavage fluid can mediate metabolism in patients with Acinetobacter baumannii ventilator-associated pneumonia
}

\author{
Bo Zhou ${ }^{1 \#}$, Manyun Guo ${ }^{2}$, Xiang $\mathrm{Hao}^{2}$, Bowen $\mathrm{Lou}^{2}$, Junhui Liu ${ }^{3 \#}$, Jianqing She ${ }^{2 \#}$ \\ ${ }^{1}$ Respiratory and Critical Care Medicine, the First Affiliated Hospital of Xi'an Jiaotong University, Xi'an, China; ${ }^{2}$ Cardiovascular Department, \\ the First Affiliated Hospital of Xi'an Jiaotong University, Xi'an, China; ${ }^{3}$ Diagnostic Department, the First Affiliated Hospital of Xi'an Jiaotong \\ University, Xi'an, China \\ Contributions: (I) Conception and design: B Zhou, J Liu, J She; (II) Administrative support: B Zhou, J Liu, J She; (III) Provision of study materials or \\ patients: B Zhou, M Guo, B Lou; (IV) Collection and assembly of data: B Zhou, X Hao, B Lou; (V) Data analysis and interpretation: M Guo, X Hao, \\ B Lou; (VI) Manuscript writing: All authors; (VII) Final approval of manuscript: All authors. \\ \#These authors contributed equally to this work as co-first authors. \\ Correspondence to: Jianqing She. Cardiovascular Department, the First Affiliated Hospital of Xi'an Jiaotong University, Xi'an, China. Email: \\ jianqingshe@xjtu.edu.cn; Junhui Liu. Diagnostic Department, the First Affiliated Hospital of Xi'an Jiaotong University, Xi'an, China. Email: \\ liu1109@xjtu.edu.cn; Bo Zhou. Respiratory and Critical Care Medicine, the First Affiliated Hospital of Xi'an Jiaotong University, Xi'an, China. \\ Email: zb_bob@stu.xjtu.edu.cn.
}

\begin{abstract}
Background: Ventilator-associated pneumonia (VAP) is a major public health problem and is most commonly caused by Acinetobacter baumannii (Ab) infection. In our study, we investigated the profiles of exosomal microRNAs (miRNAs) extracted from the bronchoalveolar lavage fluid (BALF) and serum of patients with Acinetobacter baumannii ventilator-associated pneumonia (Ab-VAP). We also examined the serum metabolomic profiles of these patients. Our aim was to study the associations between lung tissuederived exosomal miRNAs and changes in global metabolism in patients with Ab-VAP.
\end{abstract}

Methods: Consecutively sampled patients admitted to an intensive care unit (ICU) for pulmonary infection treatment were enrolled in this study. Demographic information and biochemical measurements were collected. Serum samples were obtained following overnight fasting on admission. Bronchoscopies were performed and BALF samples were collected from each patient. Exosomes were extracted using kits from System Biosciences (SBI) and miRNA sequencing was performed. Non-targeted metabolomics were used to express metabolic profiles.

Results: We found significant changes in the miRNA profiles of patients with Ab-VAP; these changes occurred in both BALF exosomal miRNA and serum exosomal miRNA. Gene Ontology analysis further identified the function of miRNA in system metabolism. Serum metabolomic profiles and ratios of biological significance were found to be differentially regulated in Ab-VAP patients. This differential regulation was correlated with the differential expression of miRNAs.

Conclusions: Our data summarizes the dysregulation of serum metabolism and exosomal miRNA excretion that occurs in Ab-VAP patients. The correlation found between BALF exosomal miRNA and dysregulated metabolism, as indicated by the irregular expression of metabolites in the cellular metabolic pathway, highlights potential biomarkers for the diagnosis and treatment of Ab infection.

Keywords: Acinetobacter baumannii; exosomes; MicroRNA profiles; metabolomics; ventilator-associated pneumonia (VAP)

Submitted Mar 10, 2020. Accepted for publication Sep 11, 2020.

doi: $10.21037 /$ atm-20-2375

View this article at: http://dx.doi.org/10.21037/atm-20-2375

(c) Annals of Translational Medicine. All rights reserved. 


\section{Introduction}

Ventilator-associated pneumonia (VAP) is a major public health concern due to the increasing morbidity and mortality rates among intensive care unit (ICU) patients (1). Acinetobacter baumannii (Ab) is the most common pathogen known to cause VAP (2). As antimicrobial resistance is prevalent in patients infected with $\mathrm{Ab}$, options for treating this infection are limited (3). Therefore, a better understanding of the pathophysiology of $\mathrm{Ab}$ infection is of great clinical significance for the development of targeted novel therapies for patients with Acinetobacter baumannii ventilator-associated pneumonia (Ab-VAP).

Previous research that examined bronchoalveolar lavage fluid (BALF) found that "exosome containers" in this fluid mediate intercellular communication in respiratory diseases (4-6). Exosomes have been shown in vivo to serve as containers that carry and rapidly release selective molecules, such as microRNAs (miRNAs) and long non-coding RNAs (lncRNAs) (7). Recent studies have also shown that exosomal miRNAs in BALF and serum exhibit distinct features in patients with asthma, acute respiratory distress syndrome, and pulmonary infection $(5,8-11)$. Exosomes transfer miRNAs to target cells, allowing these miRNAs to exert their effects on these targets (12). Patients with pulmonary infection, particularly that caused by $\mathrm{Ab}$, often exhibit systemic inflammasome activation and metabolism dysregulation (1); however, there is a lack of evidence describing the mechanisms by which BALF exosomes modulate these patients' circulatory metabolism and influence disease progression.

Metabolomics studies have shown that alterations occur in the circulatory metabolism of patients with pulmonary infection $(13,14)$. Critical pulmonary infection can cause malnutrition, gut microbiota dysregulation, and changes in energy metabolism, which can result in metabolic disturbance (15-17). Due to its persistent course, the metabolic features of $\mathrm{Ab}$ infection are well defined. However, few studies have discussed the global metabolic profiles of patients with Ab-VAP and the mechanisms by which they are dysregulated. Recent studies have shown that adipose-derived exosomal miRNAs can modulate systemic metabolism by interfering with gene expression in distant tissues, providing a novel mechanism of cell-cell crosstalk (7). However, the effect of altered BALF exosomal miRNA profiles on serum metabolite profiles during pulmonary infection has not been explored. We therefore hypothesized that BALF exosomes released from both immune and structural lung cells carry miRNAs to target organs where they exert effects that alter the serum metabolite profiles of Ab-VAP patients.

In this study, we investigated BALF exosomal miRNA profiles and serum metabolic profiles to study the associations between lung tissue-derived exosomal miRNAs and changes in the global metabolism of Ab-VAP patients. We showed that the profile of exosomal miRNAs expressed in BALF and serum, as well as the serum metabolite profiles, were significantly different in Ab-VAP patients compared with community-acquired pneumonia (CAP) patients. These altered exosomal miRNA profiles were significantly enriched in cellular metabolic pathways and notably correlated with the metabolism of alcohols, indoles, and alkylamines. This study provides new evidence that highlights how lung tissue-derived exosomal miRNAs affect systemic metabolism. We also revealed potential therapeutic targets for the treatment of Ab infection.

\section{Methods}

\section{Subjects}

Between March 2018 and September 2018, 40 patients that attended the First Affiliated Hospital of Xi'an Jiaotong University in China were enrolled in this study. Of these patients, 15 were diagnosed with VAP and 25 were diagnosed with CAP; the CAP patients were the control group. The age range of the study cohort was $50-75$ years. This study was approved by the Ethics Committee of Xi'an Jiaotong University (2018G-169), and all patients gave written informed consent according to the Declaration of Helsinki (as revised in 2013). Ab was present in the BALF collected from VAP patients; it was not present in that of CAP patients. Subjects were excluded if they met any of the following criteria: they were taking or had previously taken any drugs known to influence lipid metabolism or endocrine function; they had a chronic respiratory disease, cardiac disease, or endocrine disease; they were undergoing hemodialysis for renal failure; they had acute or chronic hepatitis with increased transaminase activities; or they had a malignant tumor. Demographic information and biochemical measurements were obtained from all subjects as described in previous studies (18-20).

\section{BALF and serum collection and isolation of exosomes}

During bronchoscopy, $20 \mathrm{~mL}$ of BALF was collected 
from each patient. Serum from participants was collected after overnight fast. The collected BALF and serum were then centrifuged at 3,000 rpm for $15 \mathrm{~min}$ to separate cells and cell debris. The supernatant was then removed. Following the manufacturer's instructions, exosomes were precipitated using System Biosciences' (SBI's) ExoQuick Kit. The precipitated exosomes were then resuspended in $100 \mu \mathrm{L} 1 \times$ phosphate-buffered saline (PBS). The size and morphology of the exosomes were examined using a Hitachi HT7700 electron microscope (21). Each preparation of exosomes was based on Minimal Information for Study of Extracellular Vesicles 2018 (MISEV2018), including quantitative measures of BALF exosomes, testing for exosome abundance (protein content), and the presence of exosome-associated components. The protein concentrations of the resuspended pellet preparations were measured by detergent compatible (DC) protein assay (BioRad Laboratories, Hercules, CA, USA) according to the manufacturer's instructions.

\section{Exosomal miRNA sequencing}

Trizol reagent $(1 \mathrm{~mL})$ was added to the exosome suspension, and RNA was extracted following the Trizol reagent manual. The RNA was precipitated in 1:1 isopropanol (v/v) and $1 \mu \mathrm{L}$ glycogen at $-20{ }^{\circ} \mathrm{C}$ overnight. An exosomal miRNA library was constructed with a QIAseq miRNA library construction kit (QIAGEN) used in accordance with the manufacturer's instructions. Libraries were sequenced on an Illumina HiSeq-2500 sequencer for 50 cycles. Reads that passed the Illumina quality filters were retained for analysis. Adapters were trimmed from the reads and reads shorter than $17 \mathrm{nt}$ were discarded. The remaining reads were then mapped to the miRBase human miRNA reference database using the FANSe3 algorithm on the Chi-Cloud NGS Analysis Platform (Chi-Biotech Co. Ltd., Shenzhen, China) (21).

\section{Serum sample preparation and biochemical measurements}

Serum samples were collected from both VAP and CAP patients on admission. Fasting venous blood was withdrawn the next morning and immediately centrifuged at 3,000 rpm for $10 \mathrm{~min}$ at $4{ }^{\circ} \mathrm{C}$. Separated serum was stored at $-80^{\circ} \mathrm{C}$ and aliquots were thawed for later processing as described in previous publications $(18,20)$. Biochemical tests, including a complete blood count, a c-reactive protein (CRP) test, a procalcitonin (PCT) test, and tests for liver and kidney function, were administered following blood collection to determine if subjects should be included or excluded in the study.

\section{Serum metabolism profile determination}

Non-targeted metabolomics profiling was performed using the XploreMET platform (Metabo-Profile Biotechnology, Shanghai, China) $(18,20)$. The sample preparation, instrumentation, metabolic annotation, and data analysis procedures were carried out as described in previous publications (22-24) with some minor modifications. The metabolites detected by this process included alcohols, aldehydes, alkylamines, amino acids, carbohydrates, fatty acids, hormones, indoles, lipids, nucleotides, organic acids, phenols, and vitamins. The ratios between metabolites correlated with biological significance were also calculated to explore the potential of dysregulated enzymes and pathways.

\section{$R N A$ isolation and real-time quantitative polymerase chain reaction (PCR) analysis}

Total RNA was isolated from the extracted serum exosomes. First-strand complementary DNA (cDNA) was generated from normalized RNA using random hexamer primers and the Superscript II kit (Invitrogen). Primers were designed by the Roche Universal Probe Library Assay Design Center. Two units of sensiFAST probe No-ROX Mix (Bioline) were used in each 96-well reaction plate (Axon). A Roche LightCycler ${ }^{\circledR} 480$ was used to perform the quantitative polymerase chain reaction (qPCR) and the real-time qPCR was repeated three times for each condition (25).

\section{Statistical analysis}

Data are presented as mean \pm standard error of the mean (SEM). for continuous variables and as a percentage for categorical variables. The miRNA reads were mapped to the miRBase human miRNA reference database using the FANSe3 algorithm and data were processed on the ChiCloud NGS Analysis Platform as described in previous publications (21). The expression levels of miRNAs in liver blood, lung blood, and peripheral blood were extracted from the Human miRNA Tissue Atlas (26). The serum metabolomics data were normalized using 
Table 1 Clinical characteristics of the patients with CAP or VAP caused by Ab Infection

\begin{tabular}{lccc}
\hline Characteristics & VAP with Ab & CAP & P \\
\hline Age (years) & $60.33 \pm 10.12$ & $67.20 \pm 6.30$ & $\mathrm{~ns}$ \\
Female & $0.33 \%$ & $0.40 \%$ & $\mathrm{~ns}$ \\
WBC $\left(\times 10^{9} / \mathrm{L}\right)$ & $10.88 \pm 1.85$ & $11.75 \pm 7.32$ & $\mathrm{~ns}$ \\
Neutrophil\% (\%) & $90.57 \pm 5.13$ & $84.70 \pm 13.09$ & $\mathrm{~ns}$ \\
CRP $(\mathrm{mg} / \mathrm{L})$ & $78.33 \pm 26.04$ & $103.98 \pm 107.85$ & $\mathrm{~ns}$ \\
PCT $(\mu \mathrm{g} / \mathrm{L})$ & $2.40 \pm 3.85$ & $0.45 \pm 0.61$ & \\
\hline
\end{tabular}

$\mathrm{Ab}$, Acinetobacter baumannii; CAP, community-acquired pneumonia; CRP, C-reactive protein; PCT, procalcitonin; WBC, white blood cell; VAP, ventilator-associated pneumonia.

MetaboAnalyst before they were analyzed as described in previous publications (27-36). Enrichment analyses were performed based on metabolic pathway-associated and drug pathway-associated metabolite sets $(37,38)$. Correlation and regression analyses of metabolites with the demographic information and biochemical measurements were calculated using Pearson's correlation (SPSS 20.0). Heatmaps and volcano plots were created using $\mathrm{R}$ studio. $\mathrm{P}$ values of $<0.05$ were considered statistically significant.

\section{Results}

BALF exosomes and serum exosomes indicated differential miRNA profiles in Ab-VAP patients compared with CAP patients

To explore whether exosomal miRNA profiles were altered in Ab-VAP patients, we collected BALF from these patients and from control CAP patients (Table 1), and performed exosome isolation using SBI kits (Figure 1A). Exosome sizes were measured by electron microscopy (Figure 1B), and we also sequenced the exosomal miRNAs. We found that miRNAs were differentially modulated in Ab-VAP patients compared with control patients. This is shown in a heatmap of the top 198 altered miRNAs (Figure 1C) and a volcano plot (Figure 1D). The top 25 downregulated miRNAs are shown in Figure $1 E$ and the top 25 upregulated miRNAs are shown in Figure $1 F$. To determine if these differential miRNAs were derived from lung tissue, we searched the Human miRNA Tissue Atlas (26), as shown in Table 2. We found that, in general, these miRNAs were highly expressed in lung tissue. This further suggests that the exosomes came from lung tissue.

\section{Significantly altered miRNA expression in BALF was correlated with serum exosome expression}

A recent study identified the systemic function of exosomal miRNAs that target gene expression in liver metabolism (7). We therefore searched the Human miRNA Tissue Atlas to determine if the top 25 miRNAs with altered profiles (26), as found in our study, were expressed in liver blood and peripheral blood. The expression levels of the top 25 differentially expressed miRNAs in liver blood, lung blood, and peripheral blood are listed in Table S2. We selected miRNAs from the Human miRNA Tissue Atlas that are expressed more abundantly in the serum exosome In line with the results of our BALF analysis, qPCR analysis of serum exosomes also showed significant differential expression of miRNAs such as miR-338-3p, miR-26b-5p, miR-30e-5p, and miR-424-5p (Figure 2A). This suggests that miRNAs excreted from lung cells can enter the circulatory system and affect distant organs.

\section{Differentially expressed miRNAs were significantly enriched in metabolic patbways}

As differentially expressed miRNAs were detected in AbVAP patients, we sought to determine if these altered miRNA profiles played a crucial role in metabolic pathways. We used KEGG pathway analysis and Gene Ontology enrichment analysis based on the top 100 differential miRNAs found in our study. KEGG pathway analysis showed enrichment of a number of molecules related to metabolic signaling pathways, including PI3K-Akt, thyroid hormone, and AMPK; these molecules were also enriched in other cancer-related and cytoskeleton signaling pathways 
A

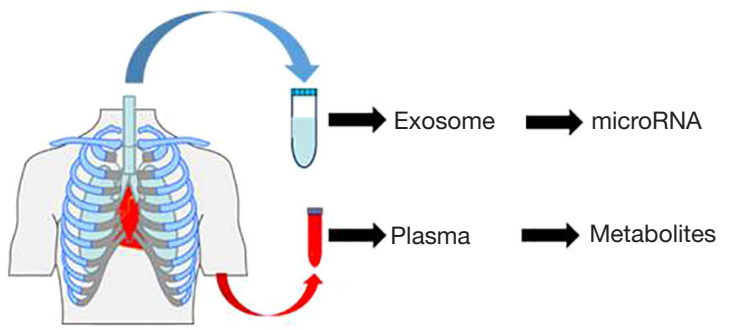

C

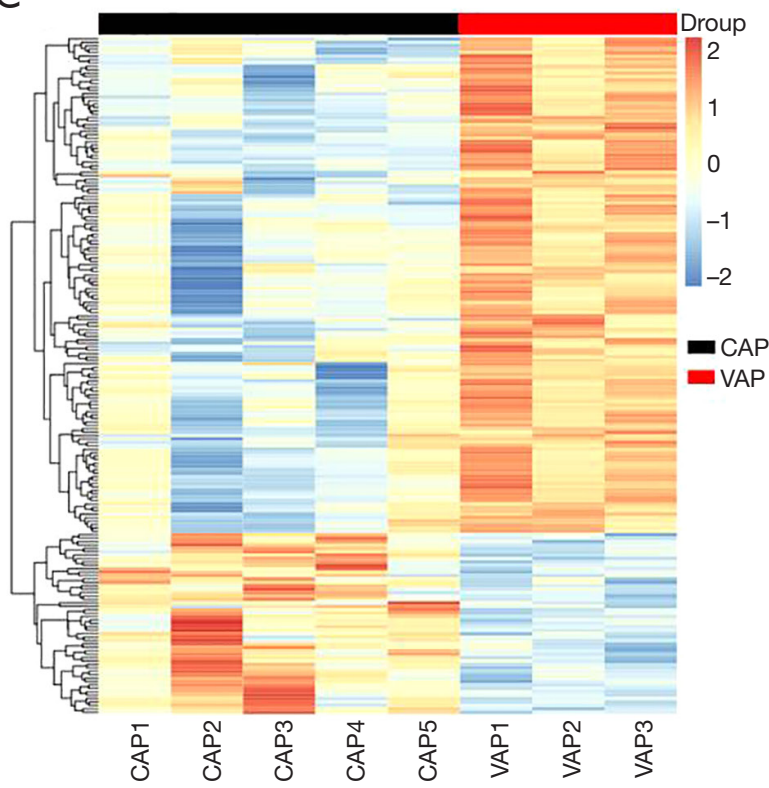

B

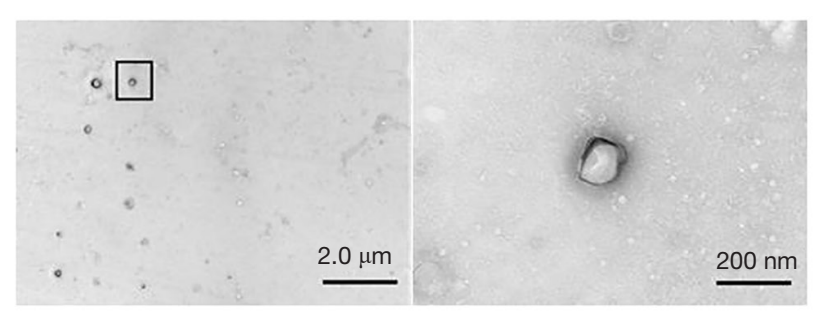

D

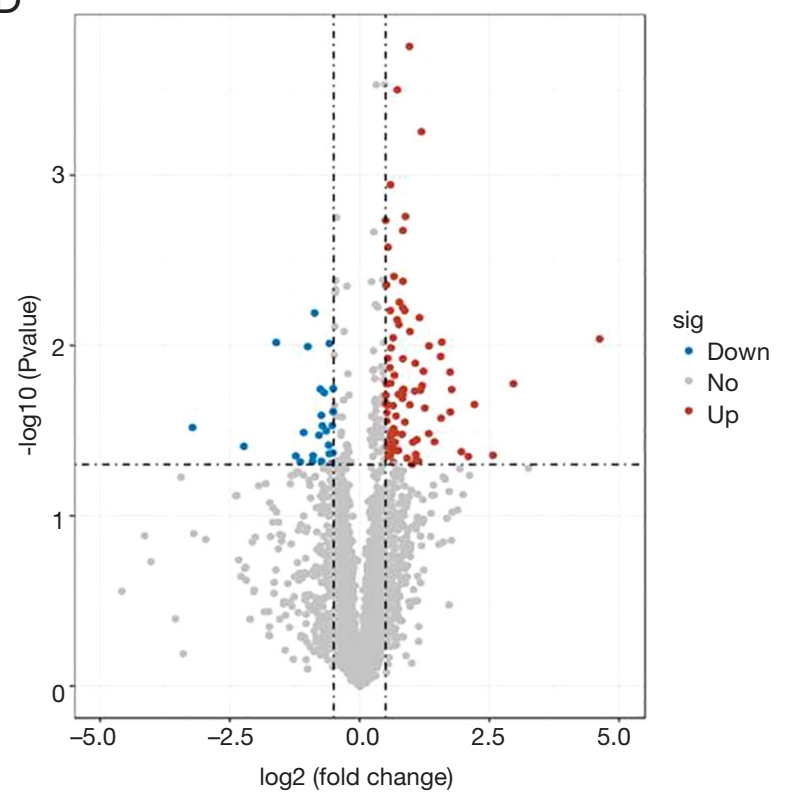

E

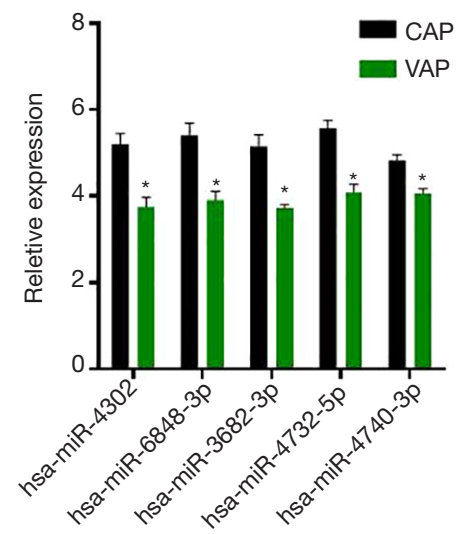

$\mathrm{F}$

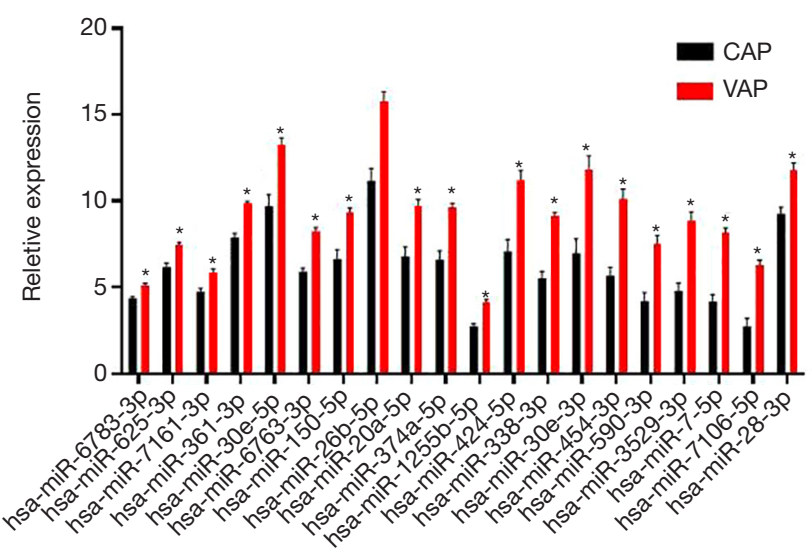

Figure 1 BALF exosomes and serum exosomes indicated differential miRNA profiles in Ab-VAP patients compared with CAP patients. (A) Study protocol for the isolation of EVs in bronchoalveolar lavage fluid and serum sample collection. (B) Characterization of bronchoalveolar exosomes using electron microscopy. Scale bar: $2.0 \mu \mathrm{m}$ and $200 \mathrm{~nm}$ respectively. (C) Heatmap showing 198 microRNAs altered in 3 VAP patients caused by $\mathrm{Ab}$ and $5 \mathrm{CAP}$ patients. (D) Volcano plot of the dysregulated microRNAs. Red dots indicated upregulated microRNAs with $\mathrm{P}<0.05$ and Fold Change $>2$ with upregulation. Blue dots indicated downregulated microRNAs with $\mathrm{P}<0.05$ and Fold Change $>2$ with down regulation. Grey dots indicated microRNAs with $\mathrm{P}>0.05$ or Fold Change $<2$. (E) Alteration of down regulated microRNAs in VAP as compared to CAP. (F) Alteration of up regulated microRNAs in VAP as compared to CAP. *, $\mathrm{P}<0.05$. 
Table 2 List of the top 25 differentially expressed microRNA and their expression in liver, long and normal blood

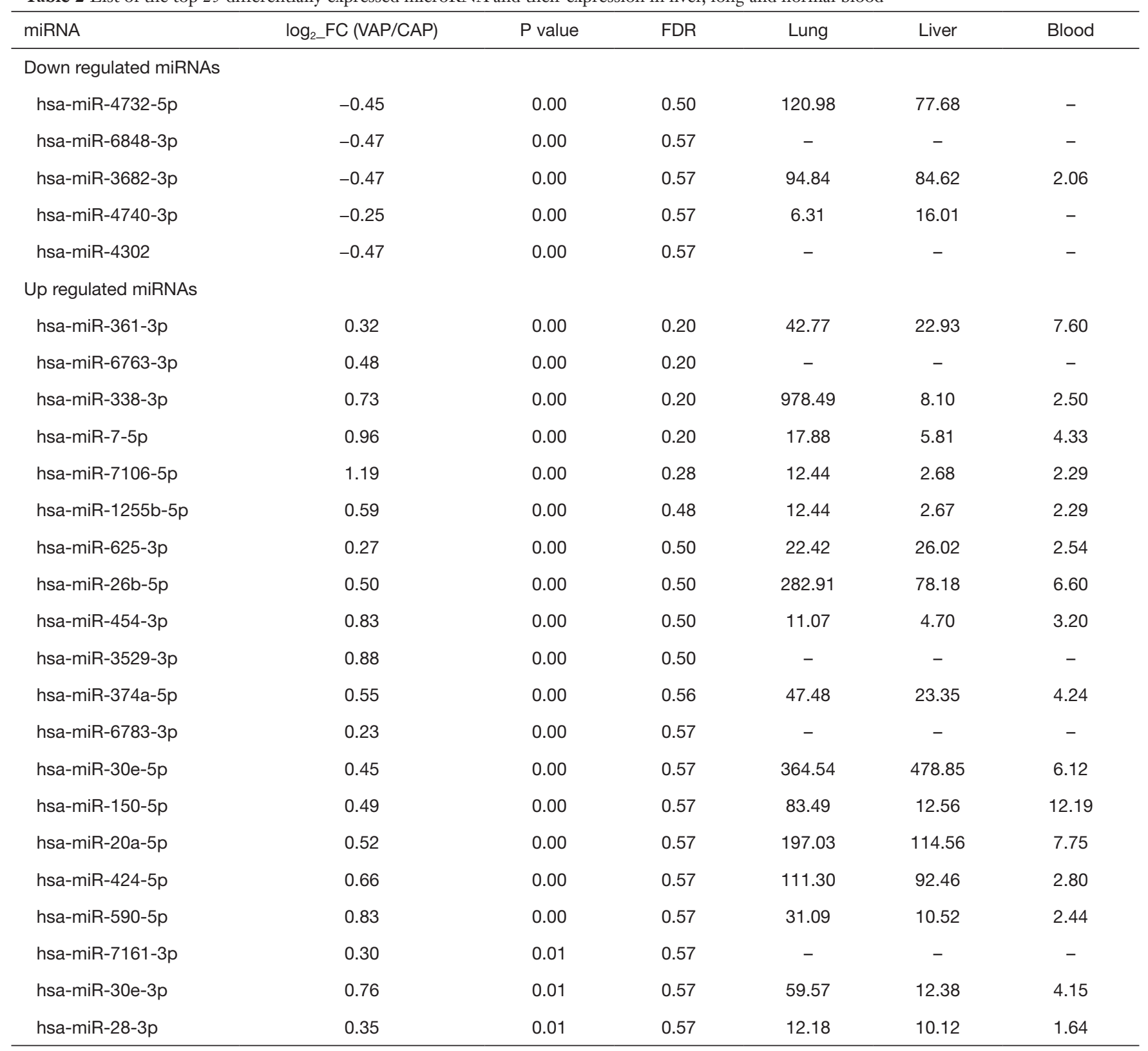

- , not found in human miRNA tissue atlas.

(Figure 3A; Table S1). Gene Ontology enrichment analysis further showed enrichment in metabolism and cell-cell crosstalk, as demonstrated by biological processes, cellular components, and molecular functions (Figure 3B; Table S2).

\section{Serum metabolites were differentially regulated in Ab-VAP patients}

Alterations in miRNA expression profiles and the results of our enrichment analysis indicated that BALF exosomal miRNA may play a role in systemic metabolism. We therefore investigated changes in the circulatory metabolic profiles of Ab-VAP patients. A total of 227 serum metabolites, including alcohols, amino acids, carbohydrates, and fatty acids, were detected in the serum of these patients. The differences in overall metabolite class values between the Ab-VAP patients and the control CAP patients are presented as a heatmap. No significant differences existed 


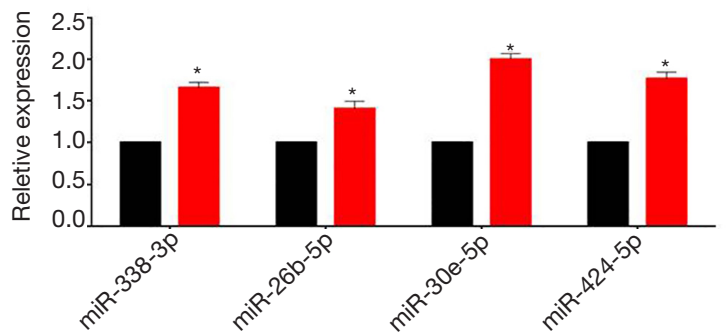

Figure 2 Significantly altered miRNA expression in BALF was correlated with serum exosome expression. (A) Relative expression of microRNAs in VAP as compared to CAP in the serum exosomes. *, $\mathrm{P}<0.05$.

between the two groups (Figure $4 A$ ).

We then compared the metabolite levels and ratios of biological significance between CAP patients and Ab-VAP patients. A volcano plot was used to show significantly altered metabolite profiles and ratios with $\mathrm{P}$ values of $<0.05$ with fold change more than twice (Figure $4 B$ ). Of these metabolites, the profiles of hydroxypropionic acid, D-Xylose, ornithine, and caproic acid (Figure 4C) were found to be significantly altered. The ratios of biological significance between L-valine and alpha-Ketoisovaleric acid, as well as that between tryptamine and L-tryptophan (Figure 4D), were also significantly altered.

\section{Serum metabolites were differentially regulated in metabolic pathway-associated metabolite sets and drug pathway-associated metabolite sets in Ab-VAP patients}

Based on the circulatory metabolic profiles described above, we further determined whether this altered metabolite expression played a role in different metabolic pathways. Enrichment analyses were performed to identify metabolite sets grouped by involvement with the same biological processes or drug-associated pathways. The strongest associations between altered metabolite expression and different biological processes were found in the urea cycle and phenylacetate metabolism (Figure 5A; Table S3). As $\mathrm{Ab}$ infections have a long duration and are resistant to antibiotics, we also analyzed the relationships between altered metabolite profiles and drug-associated pathways in the two groups. Metabolites with altered expression in AbVAP patients were shown to be involved in a number of drug pathways (Figure 5B; Table S4). These pathways are associated with cell cycle suppression through the inhibition of purine metabolism.

\section{Differential BALF exosomal miRNAs were correlated with serum metabolite levels}

As we found alterations in both BALF exosomal miRNA profiles and serum metabolite profiles in Ab-VAP patients, we then investigated the relationship between BALF miRNA and systemic metabolism. First, we analyzed the correlations between the top 25 altered BALF exosomal miRNA profiles and classes of metabolites. All 25 miRNAs were significantly correlated with serum alcohol levels, and a majority of these miRNAs were also significantly correlated with indoles; this is consistent with the alterations found in the metabolite ratios (Figure 6A). Figure $6 B$ shows lines drawn to connect metabolites and miRNAs with significant correlations in a network. These correlation analyses also confirmed that hydroxypropionic acid and ornithine were significantly correlated with several miRNAs, which indicates pathophysiological significance (Table S5). We can infer from this and our results that exosomes released from lung immune and structural cells may enter the circulatory system and carry miRNAs to the liver. Once in the liver, these miRNAs perform functions that affect serum metabolite profiles (Figure 6C). The exact mechanism by which these miRNAs achieve this, however, requires in vivo and in vitro investigation.

\section{Discussion}

Although BALF exosomal miRNAs have been shown to regulate a wide range of inflammatory processes $(4,8-11)$, their function in patients with $\mathrm{Ab}$ infection has yet to be investigated. In the present study, we found significantly altered exosomal miRNA profiles in the BALF of patients with Ab-VAP. Gene Ontology analysis further distinguished the functions of these miRNAs in systemic metabolism. We also found that the serum metabolomic profiles and ratios of biological significance were differentially regulated in patients with Ab-VAP; these changes were correlated with the differential expression of miRNAs. The differential expression pattern of exosomal miRNAs found in the lungs, liver, and circulation implies that BALF exosomes originating from immune and structural lung cells might carry miRNAs into the circulatory system to target systemic metabolism.

Although BALF has been shown to be involved in the development of asthma, lung cancer, and interstitial lung disease (4,8-11), its role in pulmonary infections remains unclear. Respiratory exosomes, nanovesicles circulating in 
A

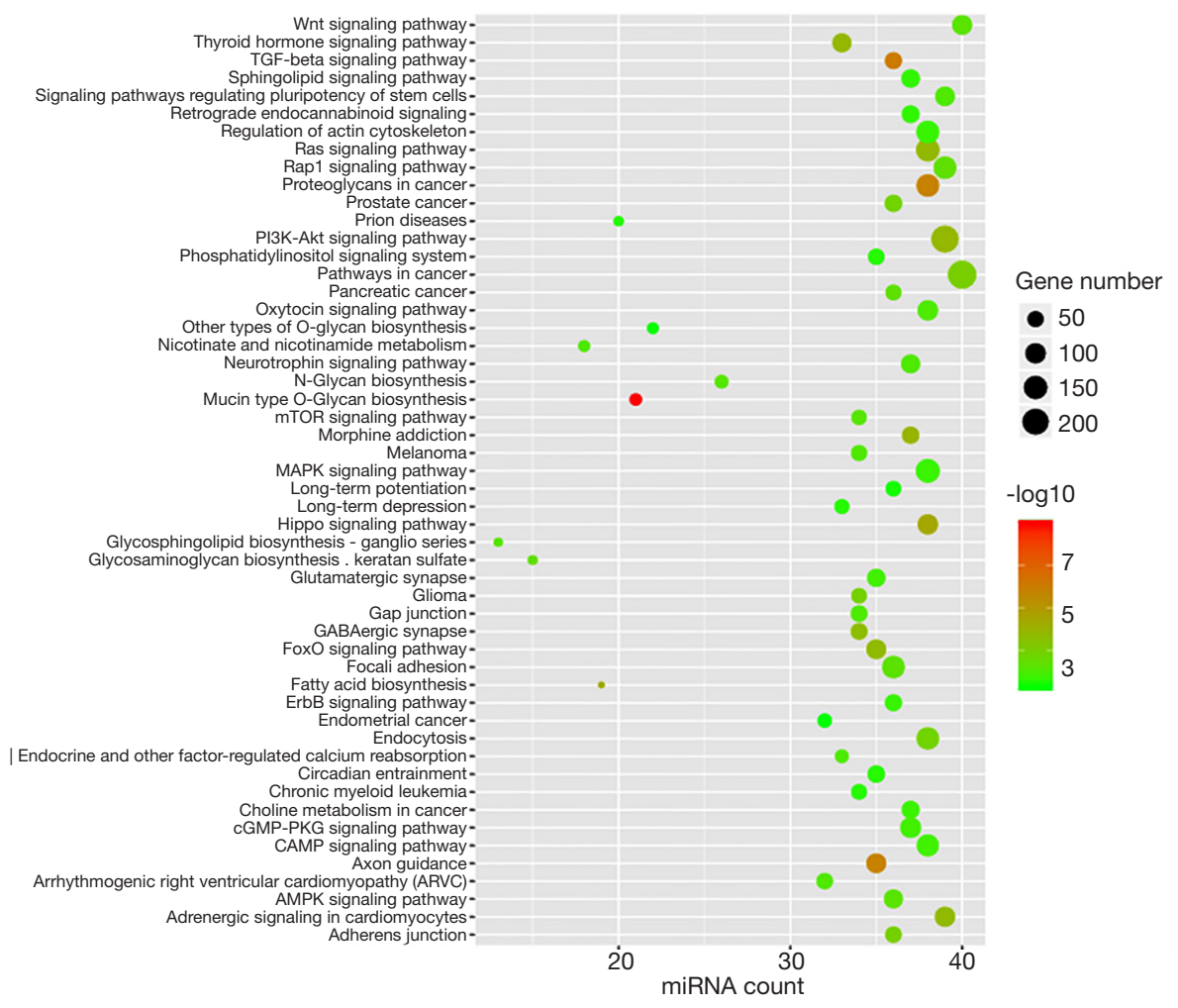

B

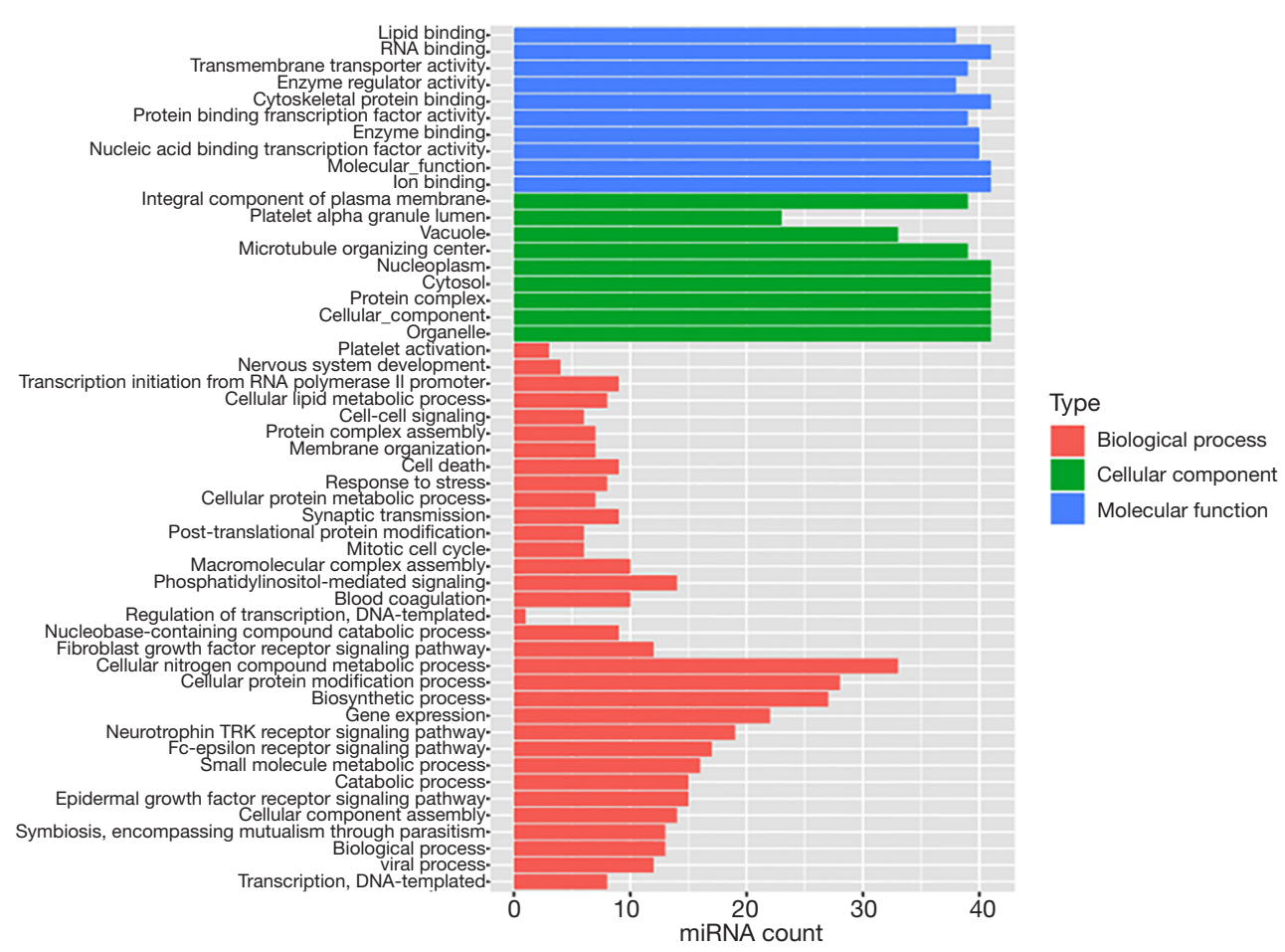

Figure 3 Differentially expressed miRNAs were significantly enriched in metabolic pathways. (A) The KEGG pathway analysis based on top 100 differential miRNAs identified significant enrichment in a number of metabolism related signaling, including PI3K-Akt, thyroid hormone, AMPK, etc. (B) The Gene Ontology enrichment analysis based on top 100 differential miRNAs revealed enrichment in metabolism and cell-cell crosstalk as displayed by biological process, cellular component and molecular function. 
A

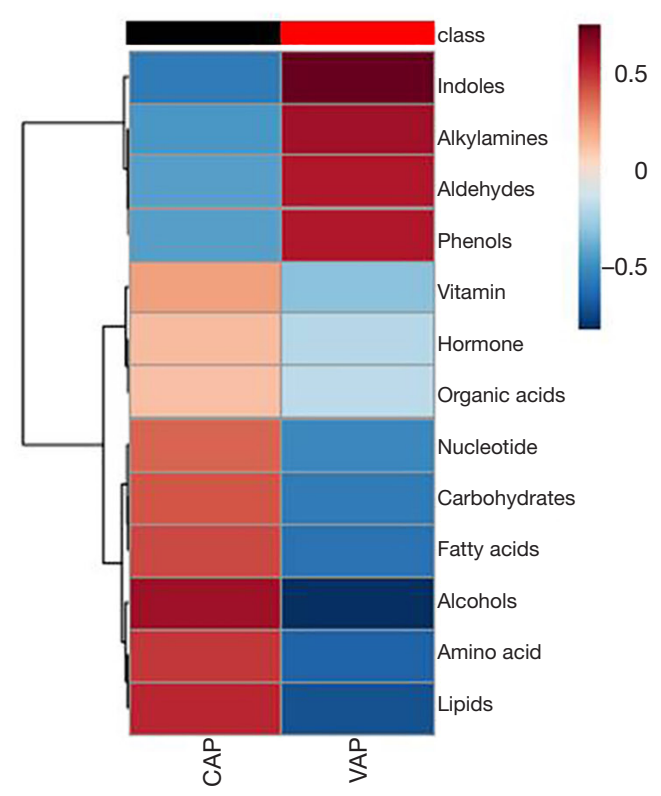

B

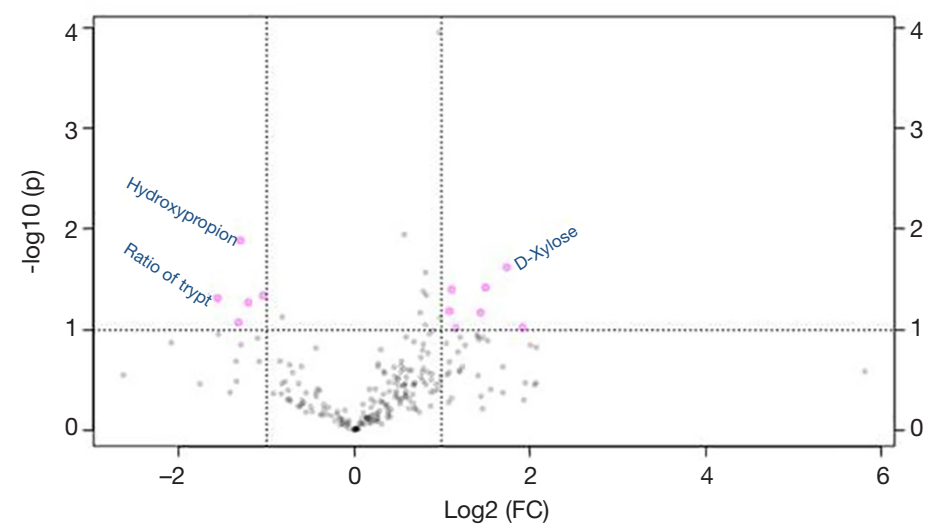

D

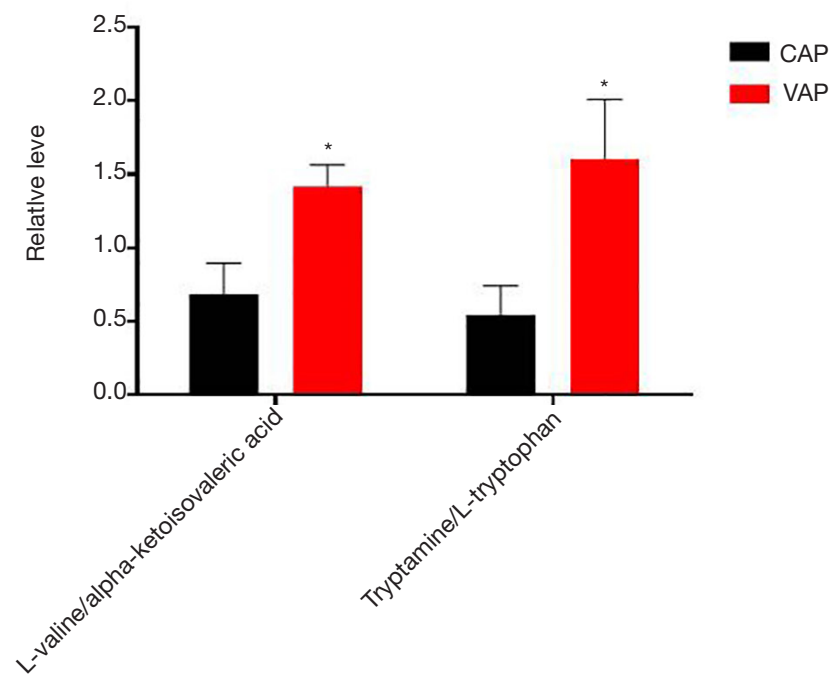

Figure 4 Serum metabolites were differentially regulated in Ab-VAP patients. (A) Heatmap showing metabolites classes between CAP and VAP with Ab infection. (B) Volcano plot of the dysregulated metabolites and ratios of biological significance. Red dots indicated upregulated metabolites with $\mathrm{P}<0.05$ and Fold Change $>2$. Blue dots indicated downregulated metabolites with $\mathrm{P}<0.05$ and Fold Change $>2$. Grey dots indicated microRNAs alteration with $\mathrm{P}>0.05$ or Fold Change $<2$. (C) Relative levels of significantly altered metabolites in VAP caused by Ab infection as compared to CAP. (D) Relative levels of significantly altered ratios of biological significance in VAP caused by Ab infection as compared to CAP. Data were analyzed using Student $t$-test. mean \pm SE. *, $\mathrm{P}<0.05$. 
A

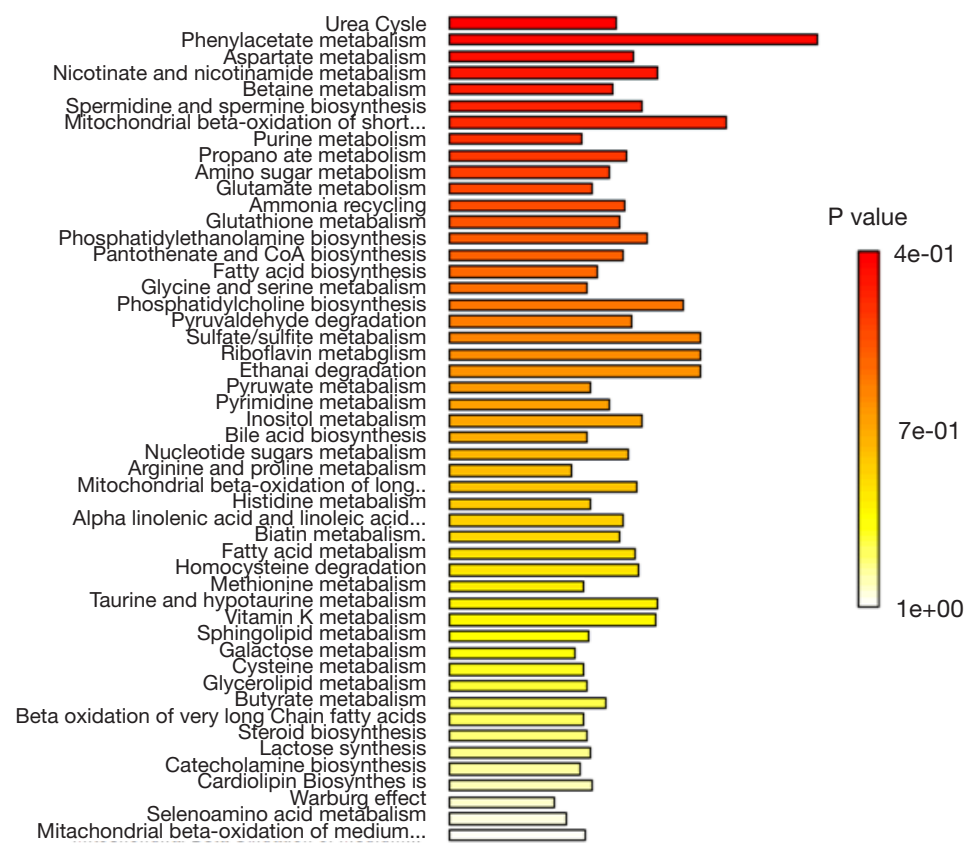

B

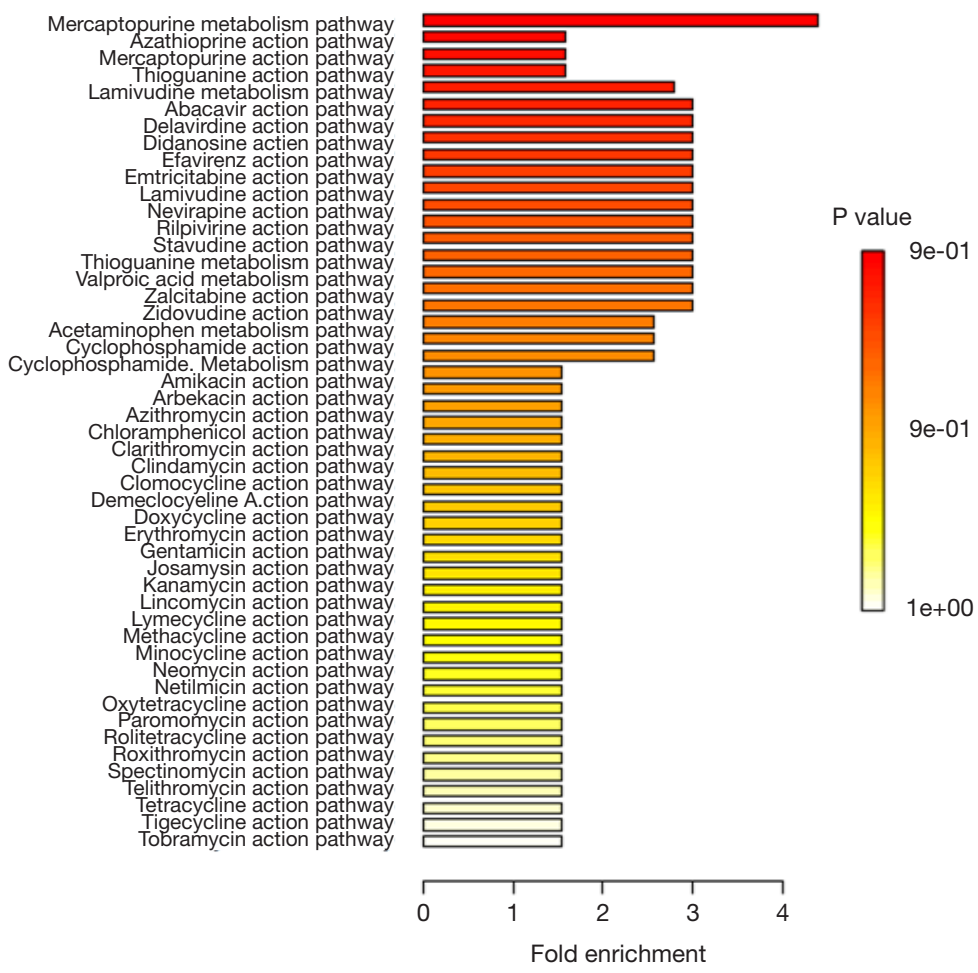

Figure 5 Serum metabolites were differentially regulated in metabolic pathway-associated metabolite sets and drug pathway-associated metabolite sets in Ab-VAP patients. (A) Enrichment analysis of metabolites based on pathway-associated metabolite sets. The strongest association was found in Urea Cycle and Phenylacetate Metabolism. (B) Enrichment analysis of metabolites based on drug-pathwayassociated metabolite sets, pointing to altered drug pathways correlating to suppressing cell cycle by inhibiting purine metabolism. 


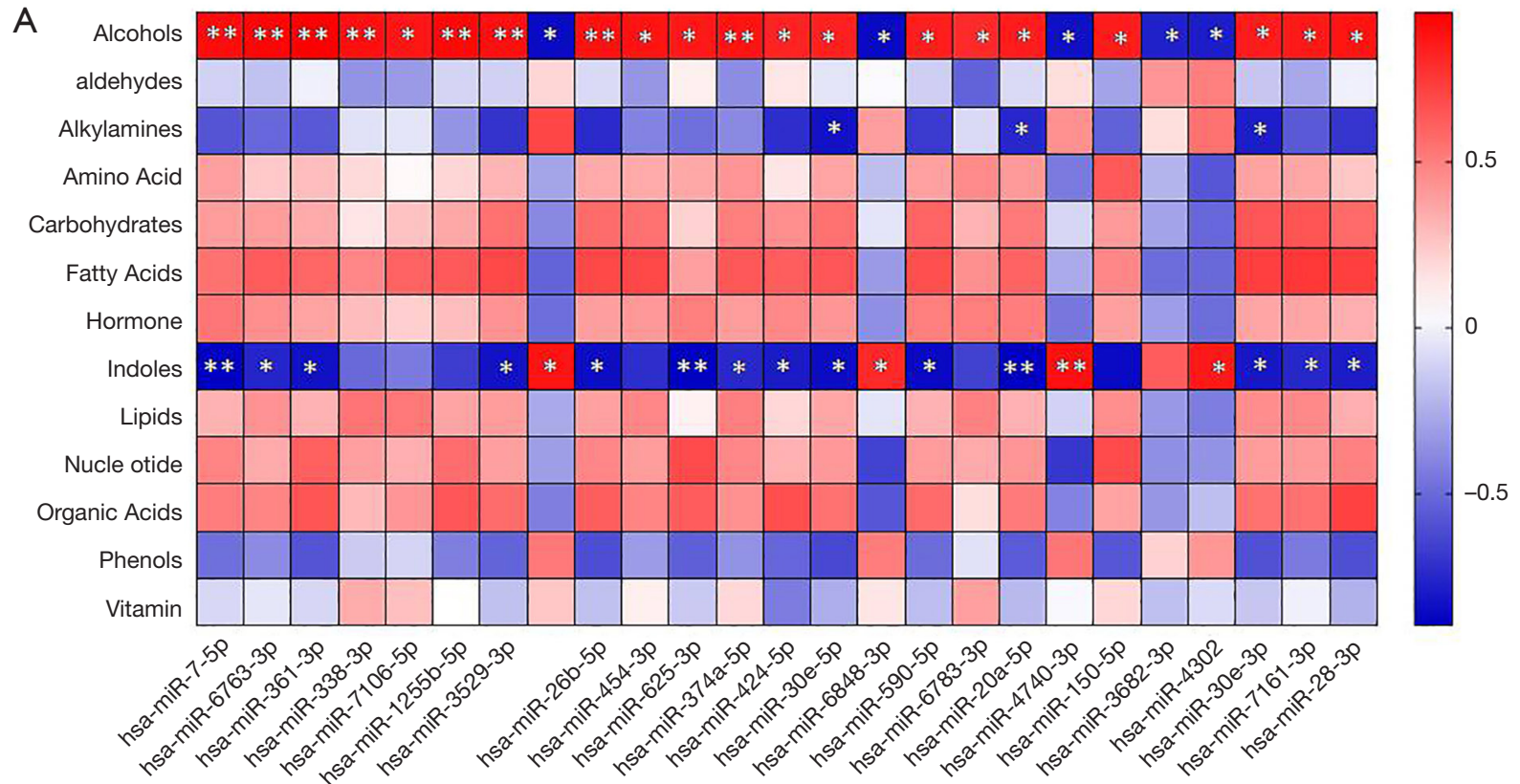

B

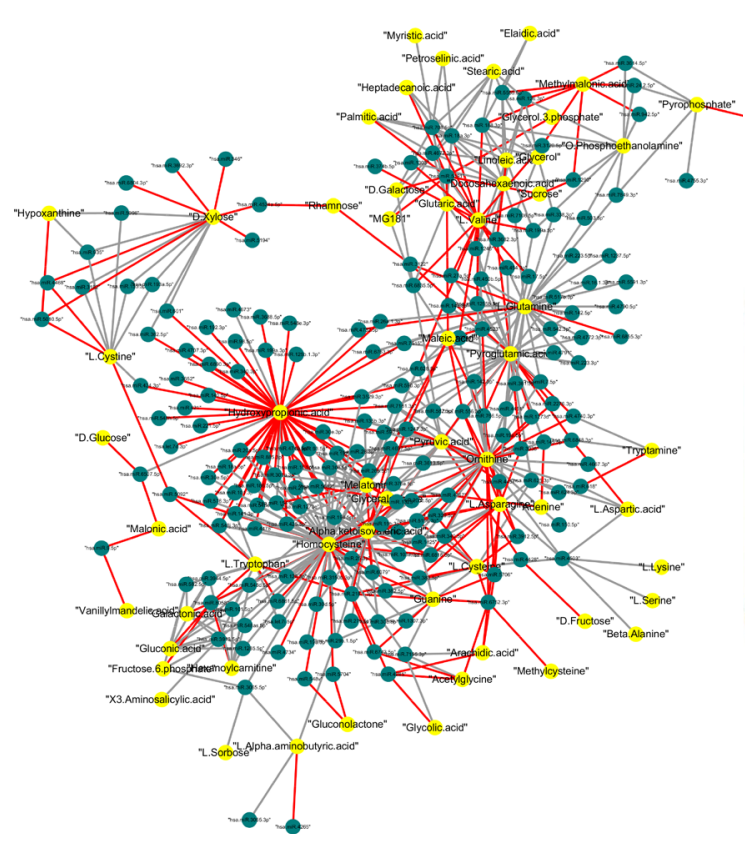

C

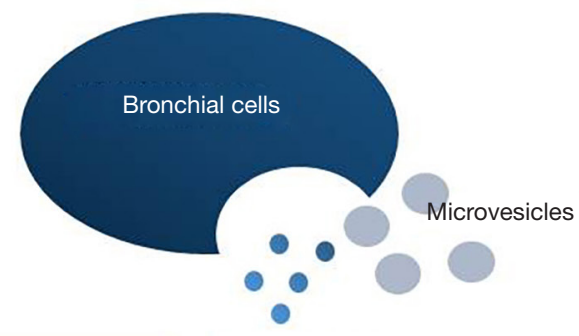

Exosomes

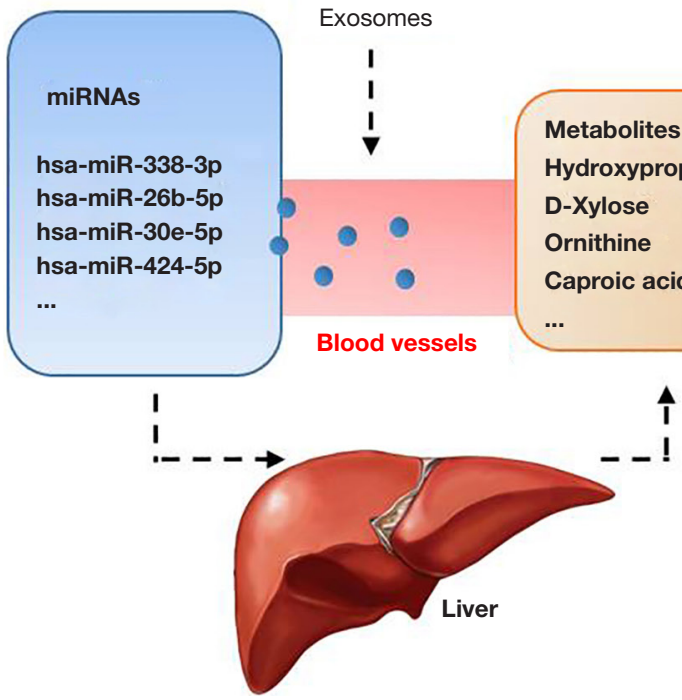

Figure 6 Differential BALF exosomal miRNAs were correlated with serum metabolite levels. (A) Correlations between BALF exosome microRNAs and serum metabolites levels. The colors in the heatmap stood for efficiency of the Pearson's correlation. *, $\mathrm{P}<0.05,{ }^{* *}, \mathrm{P}<0.01$. (B) Correlated metabolites and microRNAs identified and connected with lines in the network. (C) Schematic illustration that exosomes released from the lung tissue might enter circulation, carry microRNAs from both immune and structural cells to the liver and exert subsequent effects to serum metabolite profile. 
the respiratory tract, have been shown to be involved in the progression of inflammation-related lung damage (6). The present study examined the miRNA content of lung-derived exosomes isolated from BALF in Ab-VAP patients and CAP patients. The differential regulation of exosomal miRNAs that occurs in VAP suggests that lung-derived exosomes are involved in the propagation of this disease during $\mathrm{Ab}$ infection. The discovery of these alterations of BALF exosomal miRNA profiles provides potential biomarkers and novel insight into the underlying mechanisms of $\mathrm{Ab}$ infection, as well as the development of strategies to treat this infection.

Studies on serum metabolism have provided evidence that improves the understanding of pathophysiological mechanisms of pulmonary infectious diseases. These studies have shown that many serum metabolites could be used as biomarkers to detect metabolic disorders in patients with pneumonia $(13,14)$. Although metabolic disturbances are known to occur in pneumonia, few studies have discussed metabolic profile changes in pneumonia patients infected with Ab. Our results have shown that serum metabolism is differentially regulated during $\mathrm{Ab}$ infection. It should be noted that, in our study, only a few serum metabolites showed altered profiles in VAP patients compared with CAP patients. One reason for this could be our limited sample size. Another could be that, since both CAP and VAP are associated with systemic inflammation, circulatory metabolites might have undergone similar inflammatory changes. Whatever the cause, these metabolites that are differentially expressed in Ab-VAP have been shown to affect several drug pathways involved in the suppression of cell cycles by purine metabolism inhibition. This identifies several potential therapeutic targets for this disease, although their pathophysiological mechanisms require further exploration.

The primary novelty of our results is the notion that exosomal miRNAs derived from the lungs might enter blood circulation and regulate systemic metabolism. Consistent with these findings, we found that differentially expressed miRNAs carried in BALF exosomes are present in blood samples taken from the lungs, liver, and peripheral blood. We also found that these altered miRNA levels are correlated with changes in circulatory metabolism. This implies that BALF exosomes derived from both immune and structural lung cells might carry miRNAs into the circulatory system. Once in this system, the miRNAs are transported to target metabolism-related organs where they carry out functions that regulate the circulatory metabolism profile.

The primary limitation of the present study was that the sample size was relatively small; few patients between the ages of 50 and 75 with critical pulmonary infections met our study's inclusion criteria. Finding and recruiting CAP and VAP patients admitted to the ICU due to pulmonary infection who also have no notable medical history, including severe renal or hepatic damage, is challenging. Additionally, although we can infer from our results that lung tissue-derived exosomes might be released into peripheral blood circulation and modulate metabolism in Ab-VAP patients, further in vitro and in vivo studies are necessary to confirm both this cell-cell crosstalk function and the organs targeted by BALF exosomes.

\section{Conclusions}

The present study identified altered BALF exosomal miRNA profiles in Ab-VAP patients. These miRNAs are enriched in metabolism-related pathways and are correlated with serum metabolism changes during Ab infection. Our data implies that lung-derived exosomal miRNAs might enter the peripheral blood circulation and regulate systemic metabolism. These metabolic changes could indicate potential novel metabolic targets for the treatment of $\mathrm{Ab}$ infection.

\section{Acknowledgments}

We thank all patients involved in the study.

Funding: This study was supported by National Natural Science Foundation of China (81800390), Key Research and Development Program of Shaanxi (Program No. 2020KW-049)x, the Fundamental Research Funds for the Central Universities in China (1191329724, 191329849), and the Clinical Research Award of the First Affiliated Hospital of Xi'an Jiaotong University in China (XJTU1AFCRF-2018-025).

\section{Footnote}

Data Sharing Statement: Available at http://dx.doi. org/10.21037/atm-20-2375

Conflicts of Interest: All authors have completed the ICMJE uniform disclosure form (available at http://dx.doi. org/10.21037/atm-20-2375). The authors have no conflicts of interest to declare. 
Ethical Statement: The authors are accountable for all aspects of the work in ensuring that questions related to the accuracy or integrity of any part of the work are appropriately investigated and resolved. Written informed consent was obtained according to the Declaration of Helsinki (as revised in 2013), and was approved by the ethics committee, Xi'an Jiaotong University (2018G-169).

Open Access Statement: This is an Open Access article distributed in accordance with the Creative Commons Attribution-NonCommercial-NoDerivs 4.0 International License (CC BY-NC-ND 4.0), which permits the noncommercial replication and distribution of the article with the strict proviso that no changes or edits are made and the original work is properly cited (including links to both the formal publication through the relevant DOI and the license). See: https://creativecommons.org/licenses/by-nc-nd/4.0/.

\section{References}

1. Nair GB, Niederman MS. Ventilator-associated pneumonia: present understanding and ongoing debates. Intensive Care Med 2015;41:34-48.

2. Zilberberg MD, Nathanson BH, Sulham K, et al. A Novel Algorithm to Analyze Epidemiology and Outcomes of Carbapenem Resistance Among Patients With HospitalAcquired and Ventilator-Associated Pneumonia: A Retrospective Cohort Study. Chest 2019;155:1119-30.

3. Huang Y, Zhou Q, Wang W, et al. Acinetobacter baumannii Ventilator-Associated Pneumonia: Clinical Efficacy of Combined Antimicrobial Therapy and in vitro Drug Sensitivity Test Results. Front Pharmacol 2019;10:92.

4. Yang Y, Ji P, Wang X, et al. Bronchoalveolar Lavage FluidDerived Exosomes: A Novel Role Contributing to Lung Cancer Growth. Front Oncol 2019;9:197.

5. Lee $\mathrm{H}$, Groot $\mathrm{M}$, Pinilla-Vera $\mathrm{M}$, et al. Identification of miRNA-rich vesicles in bronchoalveolar lavage fluid: Insights into the function and heterogeneity of extracellular vesicles. J Control Release 2019;294:43-52.

6. Rollet-Cohen V, Bourderioux M, Lipecka J, et al. Comparative proteomics of respiratory exosomes in cystic fibrosis, primary ciliary dyskinesia and asthma. J Proteomics 2018;185:1-7.

7. Thomou T, Mori MA, Dreyfuss JM, et al. Adipose-derived circulating miRNAs regulate gene expression in other tissues. Nature 2017;542:450-5.

8. Moon HG, Cao Y, Yang J, et al. Lung epithelial cell- derived extracellular vesicles activate macrophagemediated inflammatory responses via ROCK1 pathway. Cell Death Dis 2015;6:e2016.

9. Maemura T, Fukuyama S, Sugita $Y$, et al. Lung-Derived Exosomal miR-483-3p Regulates the Innate Immune Response to Influenza Virus Infection. J Infect Dis 2018;217:1372-82.

10. Qiao Y, Liang X, Yan Y, et al. Identification of Exosomal miRNAs in Rats With Pulmonary Neutrophilic Inflammation Induced by Zinc Oxide Nanoparticles. Front Physiol 2018;9:217.

11. Levanen B, Bhakta NR, Torregrosa Paredes P, et al. Altered microRNA profiles in bronchoalveolar lavage fluid exosomes in asthmatic patients. J Allergy Clin Immunol 2013;131:894-903.

12. Chen J, Hu C, Pan P. Extracellular Vesicle MicroRNA Transfer in Lung Diseases. Front Physiol 2017;8:1028.

13. Chiu CY, Lin G, Cheng ML, et al. Metabolomic Profiling of Infectious Parapneumonic Effusions Reveals Biomarkers for Guiding Management of Children with Streptococcus pneumoniae Pneumonia. Sci Rep 2016;6:24930.

14. Seymour CW, Yende S, Scott MJ, et al. Metabolomics in pneumonia and sepsis: an analysis of the GenIMS cohort study. Intensive Care Med 2013;39:1423-34.

15. Brown RL, Sequeira RP, Clarke TB. The microbiota protects against respiratory infection via GM-CSF signaling. Nat Commun 2017;8:1512.

16. McFarlane AJ, McSorley HJ, Davidson DJ, et al. Enteric helminth-induced type I interferon signaling protects against pulmonary virus infection through interaction with the microbiota. J Allergy Clin Immunol 2017;140:1068-78.e6.

17. Brown RL, Clarke TB. The regulation of host defences to infection by the microbiota. Immunology 2017;150:1-6.

18. She J, Guo M, Li H, et al. Targeting amino acids metabolic profile to identify novel metabolic characteristics in atrial fibrillation. Clin Sci (Lond) 2018;132:2135-46.

19. She J, Feng J, Deng Y, et al. Correlation of Triiodothyronine Level with In-Hospital Cardiac Function and Long-Term Prognosis in Patients with Acute Myocardial Infarction. Dis Markers 2018;2018:5236267.

20. She J, Deng Y, Wu Y, et al. Hemoglobin A1c is associated with severity of coronary artery stenosis but not with long term clinical outcomes in diabetic and nondiabetic patients with acute myocardial infarction undergoing primary angioplasty. Cardiovasc Diabetol 2017;16:97.

21. Liu W, Xiang L, Zheng T, et al. TranslatomeDB: a comprehensive database and cloud-based analysis platform 
for translatome sequencing data. Nucleic Acids Res 2018;46:D206-D12.

22. Qiu Y, Cai G, Su M, et al. Serum metabolite profiling of human colorectal cancer using GC-TOFMS and UPLCQTOFMS. J Proteome Res 2009;8:4844-50.

23. Wang JH, Chen WL, Li JM, et al. Prognostic significance of 2-hydroxyglutarate levels in acute myeloid leukemia in China. Proc Natl Acad Sci U S A 2013;110:17017-22.

24. Ni Y, Qiu Y, Jiang W, et al. ADAP-GC 2.0: deconvolution of coeluting metabolites from GC/TOF-MS data for metabolomics studies. Anal Chem 2012;84:6619-29.

25. Sharma KR, Heckler K, Stoll SJ, et al. ELMO1 protects renal structure and ultrafiltration in kidney development and under diabetic conditions. Sci Rep 2016;6:37172.

26. Ludwig N, Leidinger P, Becker K, et al. Distribution of miRNA expression across human tissues. Nucleic Acids Res 2016;44:3865-77.

27. Xia J, Wishart DS. Using MetaboAnalyst 3.0 for Comprehensive Metabolomics Data Analysis. Curr Protoc Bioinformatics 2016;55:14.10.1-14.10.91.

28. Xia J, Sinelnikov IV, Han B, et al. MetaboAnalyst 3.0--making metabolomics more meaningful. Nucleic Acids Res 2015;43:W251-7.

29. Xia J, Mandal R, Sinelnikov IV, et al. MetaboAnalyst 2.0-a comprehensive server for metabolomic data analysis. Nucleic Acids Res 2012;40:W127-33.

30. Xia J, Wishart DS. Web-based inference of biological

Cite this article as: Zhou B, Guo M, Hao X, Lou B, Liu J, She J. Altered exosomal microRNA profiles in bronchoalveolar lavage fluid can mediate metabolism in patients with Acinetobacter baumannii ventilator-associated pneumonia. Ann Transl Med 2020;8(23):1561. doi: 10.21037/atm-20-2375 patterns, functions and pathways from metabolomic data using MetaboAnalyst. Nat Protoc 2011;6:743-60.

31. Xia J, Wishart DS. Metabolomic data processing, analysis, and interpretation using MetaboAnalyst. Curr Protoc Bioinformatics 2011; Chapter 14:Unit 140.

32. Xia J, Psychogios N, Young N, et al. MetaboAnalyst: a web server for metabolomic data analysis and interpretation. Nucleic Acids Res 2009;37:W652-60.

33. Xia J, Broadhurst DI, Wilson M, et al. Translational biomarker discovery in clinical metabolomics: an introductory tutorial. Metabolomics 2013;9:280-99.

34. Xia J, Sinelnikov IV, Wishart DS. MetATT: a web-based metabolomics tool for analyzing time-series and two-factor datasets. Bioinformatics 2011;27:2455-6.

35. Xia J, Wishart DS. MetPA: a web-based metabolomics tool for pathway analysis and visualization. Bioinformatics 2010;26:2342-4.

36. Xia J, Wishart DS. MSEA: a web-based tool to identify biologically meaningful patterns in quantitative metabolomic data. Nucleic Acids Res 2010;38:W71-7.

37. Frolkis A, Knox C, Lim E, et al. SMPDB: The Small Molecule Pathway Database. Nucleic Acids Res 2010;38:D480-7.

38. Jewison T, Su Y, Disfany FM, et al. SMPDB 2.0: big improvements to the Small Molecule Pathway Database. Nucleic Acids Res 2014;42:D478-84. 
Table S1 KEGG enrichment analysis of the differentially expressed microRNAs

\begin{tabular}{|c|c|c|c|}
\hline KEGG pathway & $P$ value & \#Genes & \#miRNAs \\
\hline Mucin type O-Glycan biosynthesis & 1.16E-09 & 23 & 21 \\
\hline TGF-beta signaling pathway & 0.000000939 & 60 & 36 \\
\hline Axon guidance & 0.00000153 & 94 & 35 \\
\hline Proteoglycans in cancer & 0.00000153 & 136 & 38 \\
\hline Hippo signaling pathway & 0.0000364 & 101 & 38 \\
\hline Fatty acid biosynthesis & 0.0000535 & 7 & 19 \\
\hline Morphine addiction & 0.000112304 & 63 & 37 \\
\hline Thyroid hormone signaling pathway & 0.000139178 & 84 & 33 \\
\hline PI3K-Akt signaling pathway & 0.000139178 & 224 & 39 \\
\hline Ras signaling pathway & 0.000157576 & 151 & 38 \\
\hline FoxO signaling pathway & 0.000169242 & 93 & 35 \\
\hline Adrenergic signaling in cardiomyocytes & 0.000180077 & 101 & 39 \\
\hline GABAergic synapse & 0.00023194 & 58 & 34 \\
\hline Pathways in cancer & 0.000911844 & 246 & 40 \\
\hline Adherens junction & 0.001096546 & 55 & 36 \\
\hline Glioma & 0.001104613 & 46 & 34 \\
\hline Endocytosis & 0.001402455 & 134 & 38 \\
\hline Prostate cancer & 0.001449483 & 64 & 36 \\
\hline Glycosaminoglycan biosynthesis - keratan sulfate & 0.003845853 & 12 & 15 \\
\hline Rap1 signaling pathway & 0.003845853 & 136 & 39 \\
\hline Pancreatic cancer & 0.003939949 & 49 & 36 \\
\hline Focal adhesion & 0.004893039 & 135 & 36 \\
\hline AMPK signaling pathway & 0.005127382 & 83 & 36 \\
\hline Wnt signaling pathway & 0.005198956 & 95 & 40 \\
\hline Glycosphingolipid biosynthesis - ganglio series & 0.006251308 & 10 & 13 \\
\hline mTOR signaling pathway & 0.006251308 & 44 & 34 \\
\hline N-Glycan biosynthesis & 0.00636058 & 31 & 26 \\
\hline Melanoma & 0.008399668 & 51 & 34 \\
\hline Nicotinate and nicotinamide metabolism & 0.008549548 & 20 & 18 \\
\hline Arrhythmogenic right ventricular cardiomyopathy (ARVC) & 0.008549548 & 54 & 32 \\
\hline Neurotrophin signaling pathway & 0.008549548 & 83 & 37 \\
\hline Signaling pathways regulating pluripotency of stem cells & 0.008549548 & 91 & 39 \\
\hline Oxytocin signaling pathway & 0.009078433 & 102 & 38 \\
\hline Endocrine and other factor-regulated calcium reabsorption & 0.009219605 & 31 & 33 \\
\hline Gap junction & 0.009219605 & 57 & 34 \\
\hline Glutamatergic synapse & 0.01470712 & 74 & 35 \\
\hline cGMP-PKG signaling pathway & 0.01486864 & 107 & 37 \\
\hline cAMP signaling pathway & 0.015166575 & 127 & 38 \\
\hline Choline metabolism in cancer & 0.018244195 & 69 & 37 \\
\hline MAPK signaling pathway & 0.019036457 & 160 & 38 \\
\hline ErbB signaling pathway & 0.019442331 & 59 & 36 \\
\hline Regulation of actin cytoskeleton & 0.019577433 & 137 & 38 \\
\hline Sphingolipid signaling pathway & 0.023605903 & 76 & 37 \\
\hline Retrograde endocannabinoid signaling & 0.02462814 & 68 & 37 \\
\hline Long-term depression & 0.031399414 & 40 & 33 \\
\hline Chronic myeloid leukemia & 0.031399414 & 48 & 34 \\
\hline Phosphatidylinositol signaling system & 0.031399414 & 53 & 35 \\
\hline Circadian entrainment & 0.031399414 & 64 & 35 \\
\hline Prion diseases & 0.032819541 & 13 & 20 \\
\hline Long-term potentiation & 0.041227469 & 46 & 36 \\
\hline Other types of O-glycan biosynthesis & 0.045799651 & 19 & 22 \\
\hline Endometrial cancer & 0.049107072 & 35 & 32 \\
\hline
\end{tabular}


Table S2 GO enrichment analysis of the differentially expressed microRNAs

\begin{tabular}{|c|c|c|c|c|}
\hline GO_Category & $P$ value & genes & miRNAs & CAT \\
\hline Transcription, DNA-templated & 0 & 794 & 8 & $\mathrm{BP}$ \\
\hline Viral process & 0 & 221 & 12 & $\mathrm{BP}$ \\
\hline Biological_process & 0 & 6,124 & 13 & $\mathrm{BP}$ \\
\hline Symbiosis, encompassing mutualism through parasitism & 0 & 255 & 13 & $\mathrm{BP}$ \\
\hline Cellular component assembly & 0 & 568 & 14 & $\mathrm{BP}$ \\
\hline Epidermal growth factor receptor signaling pathway & 0 & 121 & 15 & $\mathrm{BP}$ \\
\hline Catabolic process & 0 & 904 & 15 & $\mathrm{BP}$ \\
\hline Small molecule metabolic process & 0 & 1062 & 16 & $\mathrm{BP}$ \\
\hline Fc-epsilon receptor signaling pathway & 0 & 105 & 17 & $\mathrm{BP}$ \\
\hline Neurotrophin TRK receptor signaling pathway & 0 & 170 & 19 & $\mathrm{BP}$ \\
\hline Gene expression & 0 & 343 & 22 & $\mathrm{BP}$ \\
\hline Biosynthetic process & 0 & 2,113 & 27 & $\mathrm{BP}$ \\
\hline Cellular protein modification process & 0 & 1,234 & 28 & $\mathrm{BP}$ \\
\hline Cellular nitrogen compound metabolic process & 0 & 2,664 & 33 & $\mathrm{BP}$ \\
\hline Fibroblast growth factor receptor signaling pathway & $4.44 \mathrm{E}-15$ & 95 & 12 & $\mathrm{BP}$ \\
\hline Nucleobase-containing compound catabolic process & $2.76 \mathrm{E}-14$ & 385 & 9 & $\mathrm{BP}$ \\
\hline Regulation of transcription, DNA-templated & $7.34 \mathrm{E}-13$ & 157 & 1 & $\mathrm{BP}$ \\
\hline Blood coagulation & $9.06 \mathrm{E}-13$ & 197 & 10 & $\mathrm{BP}$ \\
\hline Phosphatidylinositol-mediated signaling & $9.54 \mathrm{E}-13$ & 75 & 14 & $\mathrm{BP}$ \\
\hline Macromolecular complex assembly & $3.31 \mathrm{E}-12$ & 337 & 10 & $\mathrm{BP}$ \\
\hline Mitotic cell cycle & $1.04 \mathrm{E}-09$ & 141 & 6 & $\mathrm{BP}$ \\
\hline Post-translational protein modification & $1.11 \mathrm{E}-09$ & 70 & 6 & $\mathrm{BP}$ \\
\hline Synaptic transmission & $1.60 \mathrm{E}-09$ & 182 & 9 & $\mathrm{BP}$ \\
\hline Cellular protein metabolic process & 2.07E-09 & 159 & 7 & $\mathrm{BP}$ \\
\hline Response to stress & $1.10 \mathrm{E}-08$ & 701 & 8 & $\mathrm{BP}$ \\
\hline Cell death & $1.83 \mathrm{E}-07$ & 354 & 9 & $\mathrm{BP}$ \\
\hline Membrane organization & $9.56 \mathrm{E}-07$ & 227 & 7 & $\mathrm{BP}$ \\
\hline Protein complex assembly & 2.19E-05 & 229 & 7 & $\mathrm{BP}$ \\
\hline Cell-cell signaling & 3.32E-05 & 206 & 6 & $\mathrm{BP}$ \\
\hline Cellular lipid metabolic process & $5.53 \mathrm{E}-05$ & 63 & 8 & $\mathrm{BP}$ \\
\hline Transcription initiation from RNA polymerase II promoter & 0.0001051 & 89 & 9 & $\mathrm{BP}$ \\
\hline Nervous system development & 0.0034436 & 111 & 4 & $\mathrm{BP}$ \\
\hline Platelet activation & 0.0491226 & 55 & 3 & $\mathrm{BP}$ \\
\hline Organelle & 0 & 5,442 & 41 & $\mathrm{CC}$ \\
\hline Cellular_component & $2.92 \mathrm{E}-43$ & 8,635 & 41 & $\mathrm{CC}$ \\
\hline Protein complex & $3.58 \mathrm{E}-40$ & 1,964 & 41 & $\mathrm{CC}$ \\
\hline Cytosol & $7.59 \mathrm{E}-35$ & 1,445 & 41 & $\mathrm{CC}$ \\
\hline Nucleoplasm & $5.25 \mathrm{E}-27$ & 639 & 41 & $\mathrm{CC}$ \\
\hline Microtubule organizing center & $1.57 \mathrm{E}-09$ & 248 & 39 & $\mathrm{CC}$ \\
\hline Vacuole & 0.0033698 & 148 & 33 & $\mathrm{CC}$ \\
\hline Platelet alpha granule lumen & 0.0038401 & 22 & 23 & $\mathrm{CC}$ \\
\hline Integral component of plasma membrane & 0.0221995 & 574 & 39 & $\mathrm{CC}$ \\
\hline Ion binding & $5.02 \mathrm{E}-207$ & 3,437 & 41 & MF \\
\hline Molecular_function & $1.98 \mathrm{E}-55$ & 8,591 & 41 & MF \\
\hline Nucleic acid binding transcription factor activity & $4.55 \mathrm{E}-43$ & 588 & 40 & MF \\
\hline Enzyme binding & $2.25 \mathrm{E}-37$ & 721 & 40 & MF \\
\hline Protein binding transcription factor activity & $5.47 \mathrm{E}-28$ & 289 & 39 & MF \\
\hline Cytoskeletal protein binding & $1.82 \mathrm{E}-19$ & 418 & 41 & MF \\
\hline Enzyme regulator activity & $1.52 \mathrm{E}-15$ & 433 & 38 & MF \\
\hline Transmembrane transporter activity & $1.98 \mathrm{E}-07$ & 510 & 39 & MF \\
\hline RNA binding & 0.0001394 & 846 & 41 & MF \\
\hline Lipid binding & 0.0438036 & 289 & 38 & MF \\
\hline
\end{tabular}


Table S3 Enrichment analysis of the differentially expressed metabolites based on pathway-associated metabolite sets

\begin{tabular}{lccccc}
\hline Metabolite set & Total & Hits & Statistic & P value & FDR \\
\hline Urea cycle & 29 & 12 & 33.33 & 0.00 & 0.12 \\
Phenylacetate metabolism & 9 & 2 & 72.89 & 0.00 & 0.12 \\
Aspartate metabolism & 35 & 12 & 36.73 & 0.01 & 0.12 \\
Nicotinate and nicotinamide metabolism & 37 & 4 & 41.39 & 0.01 & 0.12 \\
Betaine metabolism & 21 & 5 & 32.66 & 0.01 & 0.12 \\
Spermidine and spermine biosynthesis & 18 & 5 & 38.44 & 0.02 & 0.29 \\
Mitochondrial beta-oxidation of short chain saturated fatty acids & 27 & 2 & 55.07 & 0.03 & 0.29 \\
Purine metabolism & 74 & 14 & 26.30 & 0.03 & 0.29 \\
Propanoate metabolism & 42 & 8 & 35.39 & 0.03 & 0.29 \\
Amino Sugar metabolism & 33 & 7 & 31.96 & 0.04 & 0.29 \\
Glutamate metabolism & 49 & 13 & 28.39 & 0.04 & 0.29 \\
Ammonia recycling & 32 & 10 & 35.03 & 0.05 & 0.29 \\
\hline
\end{tabular}

Table S4 Enrichment analysis of the differentially expressed metabolites based on drug-pathway-associated metabolite sets

\begin{tabular}{lccccc}
\hline Metabolite set & Total & Hits & Statistic & P value & FDR \\
\hline Mercaptopurine metabolism pathway & 30.00 & 2.00 & 72.89 & 0.00 & 0.27 \\
Azathioprine action pathway & 92.00 & 14.00 & 26.30 & 0.03 & 0.27 \\
Mercaptopurine action pathway & 90.00 & 14.00 & 26.30 & 0.03 & 0.27 \\
Thioguanine action pathway & 91.00 & 14.00 & 26.30 & 0.03 & 0.27 \\
\hline
\end{tabular}

Table S5 Correlation analysis between serum metabolites and BALF microRNAs

\begin{tabular}{|c|c|c|c|}
\hline Metabolites & miRNAs & $P$ value & R square \\
\hline Pyroglutamic.acid & hsa.miR.7.5p & 0.004523643 & -0.909346748 \\
\hline L.Glutamine & hsa.miR.7.5p & 0.003320577 & -0.920082013 \\
\hline Ornithine & hsa.miR.7.5p & 0.008047844 & -0.885241589 \\
\hline L.Glutamine & hsa.miR.6763.3p & 0.000162536 & -0.976384059 \\
\hline Hydroxypropionic.acid & hsa.miR.6763.3p & 0.007987498 & 0.885595603 \\
\hline Pyroglutamic.acid & hsa.miR.361.3p & 0.004970386 & -0.905792465 \\
\hline L.Glutamine & hsa.miR.361.3p & 0.007688503 & -0.887372854 \\
\hline Ornithine & hsa.miR.361.3p & 0.003161223 & -0.921666119 \\
\hline Glycerol & hsa.miR.338.3p & 0.005729458 & -0.900157059 \\
\hline L.Glutamine & hsa.miR.338.3p & 0.002842403 & -0.924982092 \\
\hline Glycerol & hsa.miR.7106.5p & 0.003060743 & -0.922689137 \\
\hline L.Glutamine & hsa.miR.7106.5p & 0.005443204 & -0.902228031 \\
\hline Docosahexaenoic.acid & hsa.miR.7106.5p & 0.004195379 & -0.912091044 \\
\hline L.Valine & hsa.miR.1255b.5p & 0.009588036 & 0.876679564 \\
\hline Pyroglutamic.acid & hsa.miR.1255b.5p & 0.003558804 & -0.917794733 \\
\hline Ornithine & hsa.miR.1255b.5p & 0.006384108 & -0.895636677 \\
\hline Docosahexaenoic.acid & hsa.miR.1255b.5p & 0.00735205 & -0.889421275 \\
\hline Homocysteine & hsa.miR.3529.3p & 0.009833993 & -0.875387773 \\
\hline L.Glutamine & hsa.miR.3529.3p & 0.008270708 & -0.883947326 \\
\hline Hydroxypropionic.acid & hsa.miR.3529.3p & 0.002536394 & 0.928376863 \\
\hline L.Glutamine & hsa.miR.4732.5p & 0.005494337 & 0.90185353 \\
\hline Hydroxypropionic.acid & hsa.miR.4732.5p & 0.0094514 & -0.877405461 \\
\hline Homocysteine & hsa.miR.26b.5p & 0.00410986 & -0.912826408 \\
\hline Ornithine & hsa.miR.26b.5p & 0.009564683 & -0.876803205 \\
\hline Hydroxypropionic.acid & hsa.miR.26b.5p & 0.002636288 & 0.927243478 \\
\hline L.Valine & hsa.miR.454.3p & 0.007708364 & 0.887253571 \\
\hline Pyroglutamic.acid & hsa.miR.454.3p & 0.001965061 & -0.935427835 \\
\hline L.Glutamine & hsa.miR.454.3p & 0.008823069 & -0.880824015 \\
\hline L.Aspartic.acid & hsa.miR.625.3p & 0.007860216 & -0.88634739 \\
\hline
\end{tabular}

